\title{
EM Modeling for GPIR using 3D FDTD Modeling Codes
}

\author{
Scott D. Nelson
}

This paper was prepared for Univeristy of California Lawrence Livermore National Laboratory DSED Home Page .

June 1995

October 1994

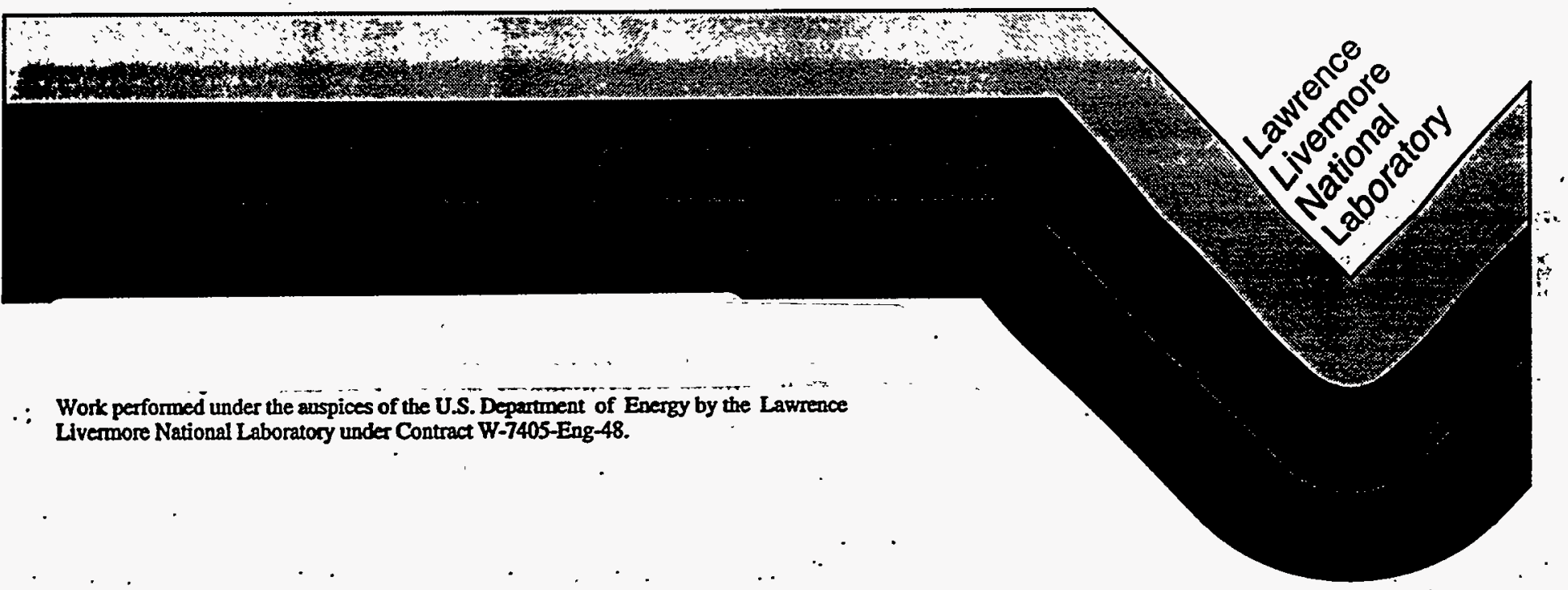




\section{DISCLAIMER}

This report was prepared as an account of work sponsored by an agency of the United States Government. Neither the United States Government nor any agency thereof, nor any of their employees, make any warranty, express or implied, or assumes any legal liability or responsibility for the accuracy, completeness, or usefulness of any information, apparatus, product, or process disclosed, or represents that its use would not infringe privately owned rights. Reference herein to any specific commercial product, process, or service by trade name, trademark, manufacturer, or otherwise does not necessarily constitute or imply its endorsement, recommendation, or favoring by the United States Government or any agency thereof. The views and opinions of authors expressed herein do not necessarily state or reflect those of the United States Government or any agency thereof. 


\section{DISCLAIMER}

Portions of this document may be illegible in electronic image products. Images are produced from the best available original document. 


\section{EM modeling for GPIR using 3D FDTD modeling codes}

Scott D. Nelson

Defense Sciences Engineering Division

Electronics Engineering

Lawrence Livermore National Laboratory

Livermore, California, 94550

\section{ABSTRACT}

An analysis of the one-, two-, and three-dimensional electrical characteristics of structural cement and concrete is presented. This work connects experimental efforts in characterizing cement and concrete in the frequency and time domains with the Finite Difference Time Domain (FDTD) modeling efforts of these substances. These efforts include Electromagnetic (EM) modeling of simple lossless homogeneous materials with aggregate and targets and the modeling dispersive and lossy materials with aggregate and complex target geometries for Ground Penetrating Imaging Radar (GPIR). Two- and three-dimensional FDTD codes (developed at LLNL) where used for the modeling efforts.

The purpose of the experimental and modeling efforts is to gain knowledge about the electrical properties of concrete typically used in the construction industry for bridges and other load bearing structures. The goal is to optimize the performance of a high-sample-rate impulse radar and data acquisition system and to design an antenna system to match the characteristics of this material. Results show agreement to within $2 \mathrm{~dB}$ of the amplitudes of the experimental and modeled data while the frequency peaks correlate to within $10 \%$ the differences being due to the unknown exact nature of the aggregate placement.

Keywords: 2D 3D FDTD electromagnetic GPIR modeling experiments dispersive concrete clutter

\subsection{INTRODUCTION}

The Lawrence Livermore National Laboratory (United States Department of Energy) has worked for many years to build unique tools and resources in the areas in electromagnetic modeling and microwave/RF antenna design and characterization. It is the unique connectivity at the Laboratory between EM modeling, design, construction, experiments, systems analysis, and field work that allows the lab to 
work in many areas in sensing systems. The following shows results from work applied to GPIR using 3D FDTD codes. These codes were used to model antenna/material interactions, discrete aggregate particles, lossy/dispersive materials, and complex target geometries. Many capabilities were used in the process of performing this work which include the areas of unique EM modeling codes, RF design and component construction, microwave / RF measurements and device characterization, and systems analysis.

The Ground Penetrating Imaging Radar Project investigated the feasibility of designing a radar system to examine the internal details of concrete structures at highway speeds such as those found in the bridge and highway industry. The central project involved the coordination of the electromagnetic modeling, imaging, EM code design and EM experimentation areas in the Laboratory to design the hardware/software system. The modeling effort generated data for the imaging effort and enabled the precise control of individual parameters in the model. Modeling also supported the experimental effort to investigate the antennas and pulsers used in the experimental hardware setup.

The modeling effort consisted of three phases: (1) verification of embedded scattering models to uniform theoretical predictions; (2) analysis of the physical antenna models used in the project and modeling effort; (3) 3D modeling of the configuration with typical targets, antennas, and concrete.

\subsection{COMPUTATIONAL ELECTROMAGNETICS CODES}

The Laboratory has for many years been working to develop EM modeling codes for use in a broad array of applications. These codes include full 2D and 3D time and frequency models which include propagation, scattering, dispersive/lossy materials, thin wires, mutual coupling, and complex and arbitrary geometries. These modeling codes include NEC [1](used for decades as THE thin wire code in many applications, both governmental and private); AMOS [2.3] (a 2.5D code used for accelerator design, antenna characterization, and radar systems design); and TSAR [4] (a 3D code used for pulse propagation, materials interaction, and antenna design). There are also several new codes under development at the laboratory that surpass the capabilities of these existing codes. The important point to note is that the existing codes have many man-decades of development effort, are well tested and verified with theory and experiments [5], and have been used for many broad ranging applications. The new codes will surpass those capabilities but are still considered research codes.

2D and 3D models were constructed and the results were compared with experimental and uniform theoretical data. The 2D data served as a preliminary test of the scattering models used in the AMOS 2.5D FDTD EM modeling code. The 3D data generated via the $3 \mathrm{D}$ code served as a verification for the 3D concrete model. The $2 \mathrm{D}$ and 3D methods were used to create time domain waveforms for the image reconstruction algorithms [6]. The use of the 3D code was necessary in order to simulate the complex target geometries used in the problem.

The 2D comparison showed good agreement between 1) the theoretical results for a 
uniform random distribution of targets and 2) the FDTD model for the effective material parameters. Later 2D and 3D models then introduced the concrete and realistic targets into the problem. These results were then used to generate data for the imaging teams and to resolve hardware and design issues.

\subsection{Code geometries}

The following diagram briefly compares the geometries of some of the codes developed and being used at the Laboratory:
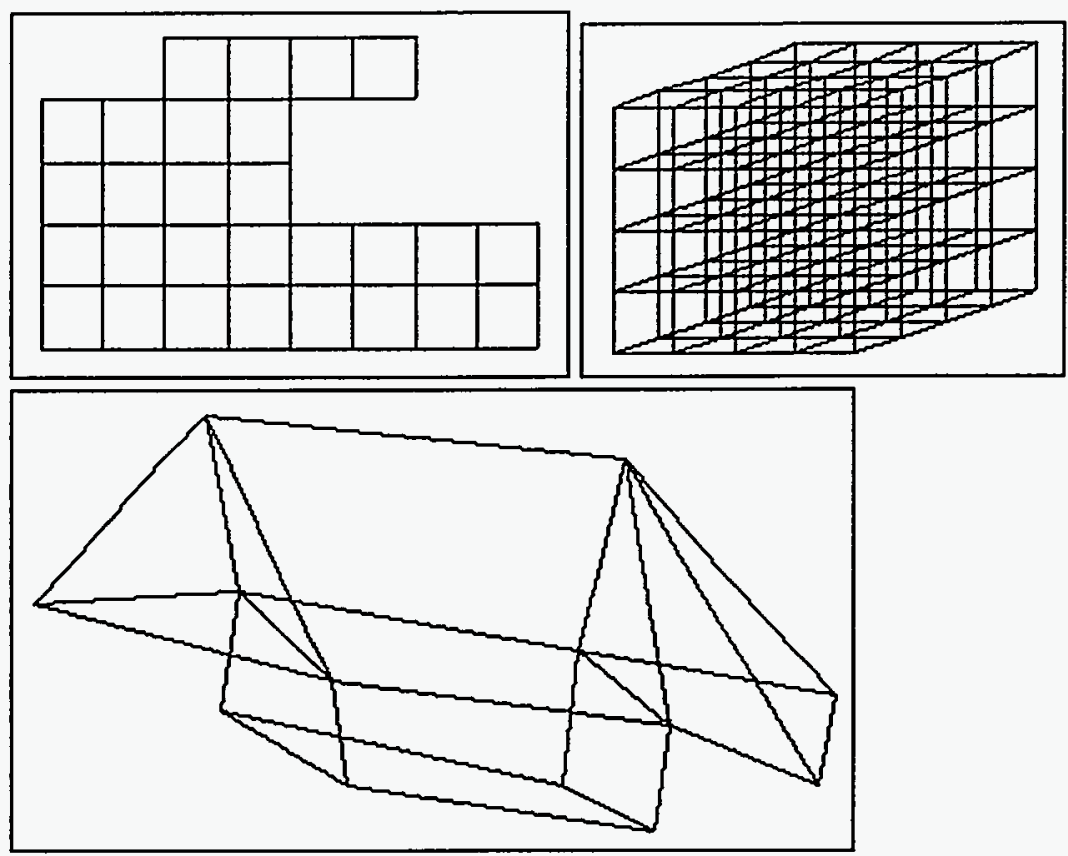

Figure 1. AMOS 2.5D coaxial cell mesh (a), TSAR 3D cubical cell mesh (b), and DSI3D arbitrary cell mesh (c) show the differences between the code's geometries.

The type of application drives which of the above codes are used. The NEC wire and surface code is used for large unbounded structures while the AMOS code is used for axisymmetric or 2D problems; especially when complex material models [5] are involved. The TSAR 3D code is used for generic problems for pulsed and CW excitation while the DSI3D [8] code is used for highly curved and space filling problems. It should be stressed that unlike other codes, these are general EM codes; the geometry determines which is appropriate for the problem.

\subsection{D CLUTTER VERIFICATION EFFORT}

A region composed of scattering bodies was modeled in 2D to compare with the Maxwell-Garnet mixing formula [9] which is the real part of 


$$
\epsilon_{e f f}=\epsilon\left\{\frac{1+\frac{2 f_{s}\left(\epsilon_{s}-\epsilon\right)}{\epsilon_{s}+2 \epsilon}}{1-\frac{f_{s}\left(\epsilon_{s}-\epsilon\right)}{\epsilon_{s}+2 \epsilon}}+j 2 f_{s} k^{3} a^{3}\left|\frac{\frac{\epsilon_{s}-\epsilon}{\epsilon_{s}+2 \epsilon}}{1-\frac{f_{s}\left(\epsilon_{s}-\epsilon\right)}{\epsilon_{s}+2 \epsilon}}\right|^{2}\right\}
$$

where er is the permittivity of the materials, $\mathrm{f}$ denotes the fill ratio of the scatterers, the s subscript corresponds to the scatters, and $a$ is the scatterer size. The form used in the comparison is

$$
\epsilon_{e_{r}} \epsilon_{0}=\frac{\epsilon_{8} \epsilon_{0}+\epsilon_{0}^{2}+f \epsilon_{s} \epsilon_{0}-f \epsilon_{0}^{2}}{\epsilon_{8}+\epsilon_{0}-f \epsilon_{g}+f \epsilon_{0}}
$$

The region of interest is composed of uniform objects with $10 \%, 30 \%$, and $50 \%$ fill ratios using dielectric aggregate. These values were chosen to span those aggregate concentrations seen in concrete. In this case the targets in the model space were oriented in the same direction and are randomly separated as show in Fig 2.

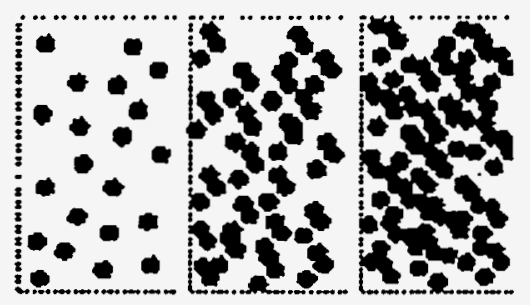

Figure 2. The packing geometries that were used (10\%, 30\%, 50\% density) to check the model results against the mixing formula.

This simulation addressed the issue of target/clutter size versus pulse width. The general rule seen earlier [7] is that targets less than one-third pulsewidth in diameter will scatter expanding spherical waves. In performing this series of $2 \mathrm{D}$ verification measurements, this relationship was again confirmed. It was also necessary to justify the packing density checkerboard that was used in the model since a finite difference square-cell grid was used. In this case, a random cellular offset was used to avoid resonances formed by rows of equally spaced clutter. 


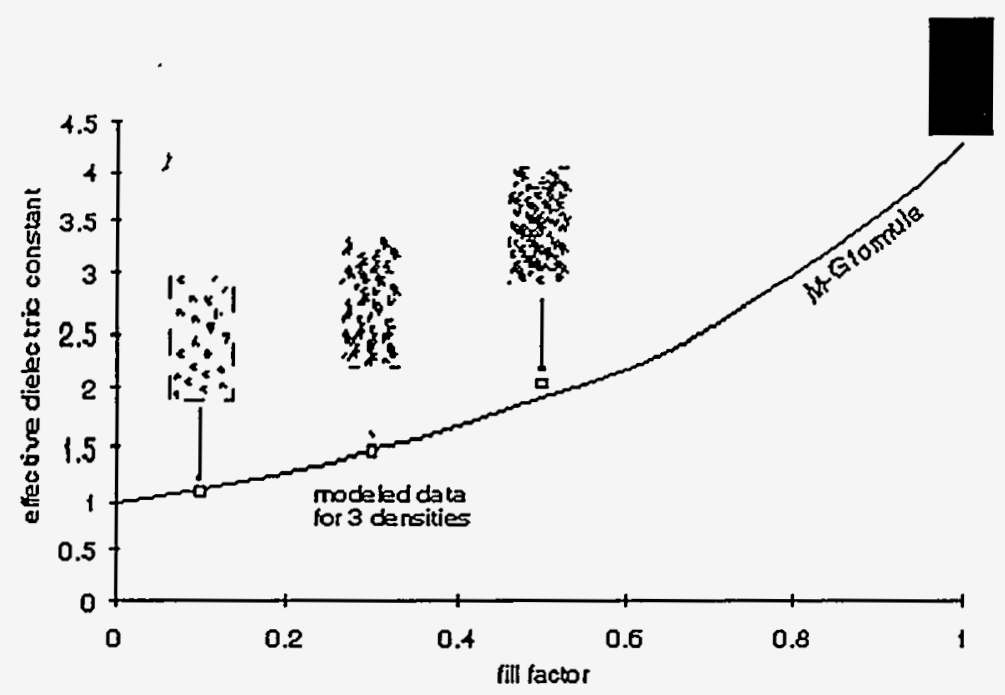

Figure 3. The results of the EM model at $10 \%, 30 \%, 50 \%$ overlaid on the MG mixing formula curve.

When the discrete aggregate models dramatized above are incorporated into the EM models with targets and layer boundaries, then typical results are generated such as those shown in Fig 4. In this case, the transmitting antenna launches an EM wave in the air space above the first layer. The energy then reflects from and propagates through the first layer (asphalt). Notice that a 90ø beamwidth, was used on the transmitting patch to represent a realistic antenna. More developed models included physical antenna structures and realistically contoured targets. The later time reflections from the asphalt/concrete interface can be seen followed by the interaction with, diffraction around, and scattering from the targets. In this case, rectangular cross sectioned targets were used to maximize the scattering back to the receiver array (not shown).
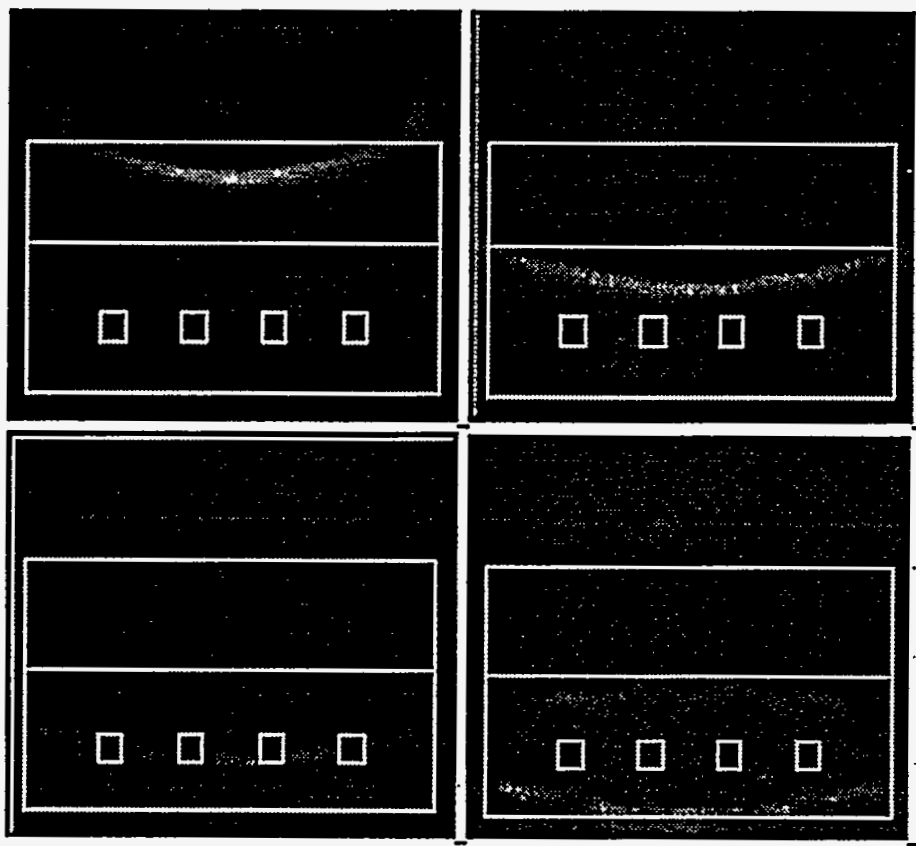

(110k:movie)

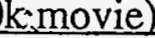


Figure 4. Four snapshots in time showing electromagnetic wave propagation in a simple, configuration. The interaction with the aggregate, targets, and layered media is clearly visible. The air, asphalt, and concrete layers are outlined as is the target grid. The aggregate apparent is visible in the concrete. The color changes are due to auto wave tracking for visualization purposes.

Additional parametric studies were performed to bound the problem from an imaging standpoint. This study included the following:

pulse width: $100 \mathrm{ps}$ to $1000 \mathrm{ps}$

target depth: $10 \mathrm{~mm}$ to $2 \mathrm{~m}$

target size: $5 \mathrm{~mm}$ to $100 \mathrm{~mm}$

aggregate $\%: 10 \%$ to $50 \%$

receiver: $5 \mathrm{~mm}$ to $45 \mathrm{~mm}$ grid spacing

multi-target: single and multi- targets for grate simulations

layers: 1,2 , and 3 layers

and the results can be seen in the previous imaging report[6].

\subsection{EXPERIMENTAL FACILITIES}

The Laboratory has unique capabilities and expertise in designing and testing a broad range of devices. Many of these facilities were used in the process of completing this series of modeling and experimental activities:

1. TEM cells - for small and large devices, to cover the $5 \mathrm{~Hz}+$ range of the spectrum. These are useful for low frequency coupling measurements or characterization measurements on small calibration probes or devices.

2. Mode Stir Chamber - presently used as a research device in studying the effects of complex modal configurations on (usually) enclosed equipment. This facility is also studying the criteria needed to simulate real world coupling scenarios.

3. Anechoic Chamber - used in the $1-30 \mathrm{GHz}$ range for studying coupling issues, designing and characterizing antennas and antenna systems, and measuring complex scattering targets. This facility was recently modified to allow for monopulse operation in the sub-nanosecond regime. 


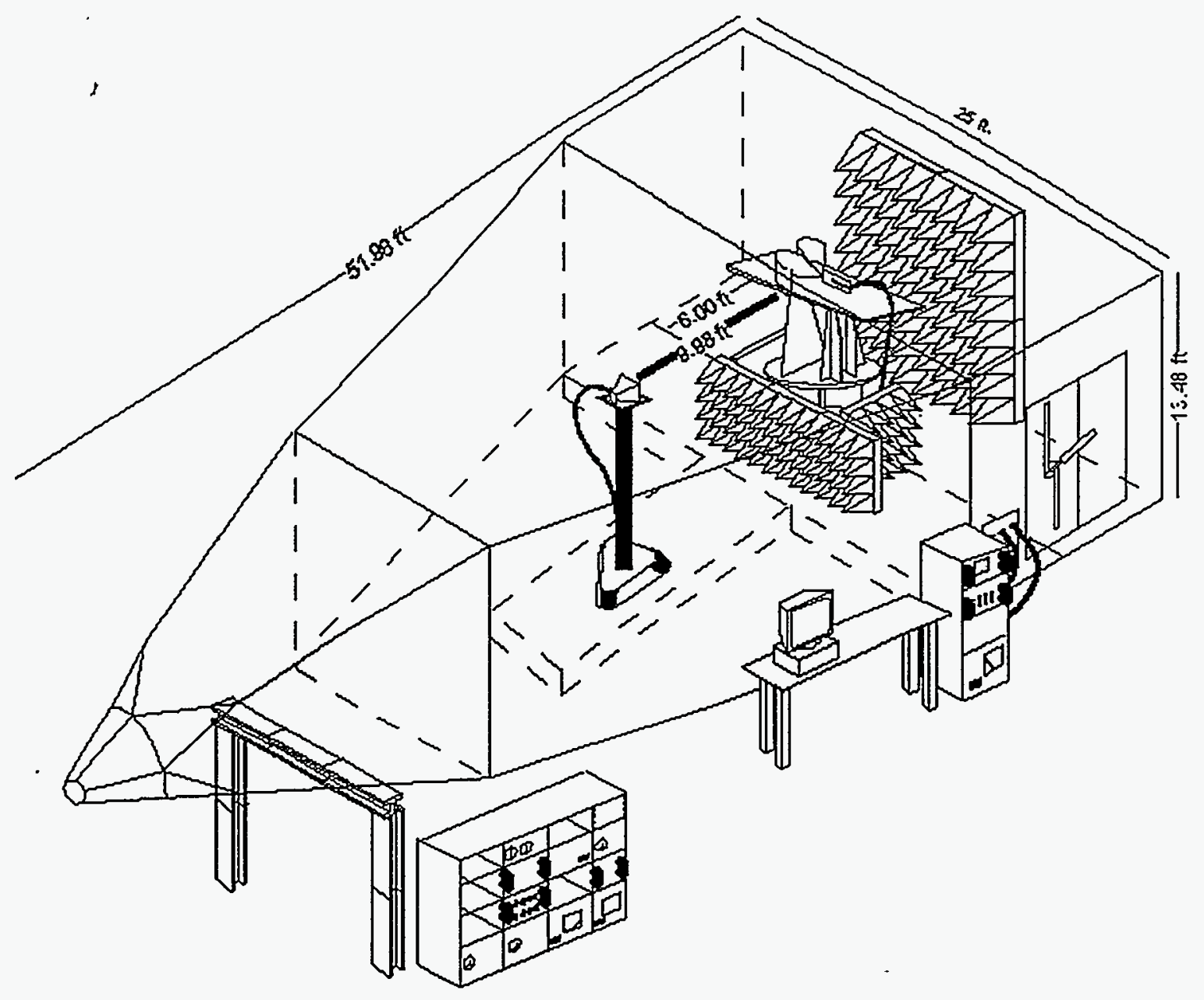

Figure 5. The anechoic chamber and it's associated control systems and RF sources can be seen. Note the GPIR project's antenna in the facility undergoing pattern tests.

4. EMPEROR monocone facility - used in the $150 \mathrm{MHz}+$ range for both low power and high power $\mathrm{CW}$ measurements and pulsed measurements to characterize devices in the intermediate frequency regions. This facility is calibrated and absolute gain measurements are possible.

5. Injection test station - - used for device and component testing for coupling and susceptibility in low and high power environments.

6. Multi-band high power source -- used primarily with the Anechoic Chamber to characterize systems or to produce internal coupling measurements in the $1-18 \mathrm{GHz}, 200 \mathrm{~W} \mathrm{CW}$, range.

7. Material characterization equipment for complex permittivity measurements [10] on small samples. 


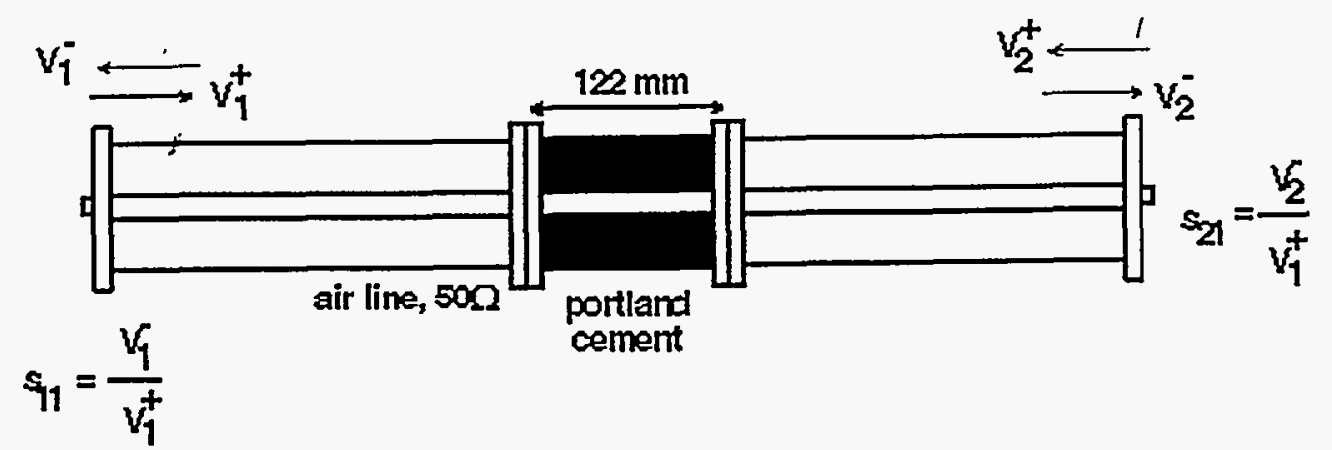

Figure 6. The coaxial air line used in the material characterization experiments shows the equipment dimensions and the material placement.

\subsection{EXPERIMENTS}

The following list (for completeness) is presented to give the reader an idea of the processes involved in performing the EM modeling and experimental design and verification efforts at the Laboratory in the EM arena as part of the GPIR or other programs:
Antenna design
Radar system design
chirped/CW/impulse radar
Circuit design
RF device characterization
RF coupling measurements
Fast pulse switching
High power $\mathrm{CW}$ measurements
Signal analysis
High voltage pulse generators
Systems integration
Materials characterization
RF susceptibility
High current generators
Antenna characterization
Field tests
EM radiation efficiency, cross section
EM modeling
Accelerator modeling
Antenna modeling
$\mathrm{RF}$ devise modeling
Test facilities design and modeling

\subsection{Material characterization}

As was mentioned in Section 3, the complex material permittivity models were used 
in the 2D and 3D EM modeling portion of the project. These models were based on the characteristics of the cement and the aggregate composing the concrete. The scattering ([s]) parameters of the cement were measured during curing in the coaxial line and the complex permittivity was extracted [10]. This series of curves is reproduced here for the convenience of the reader:

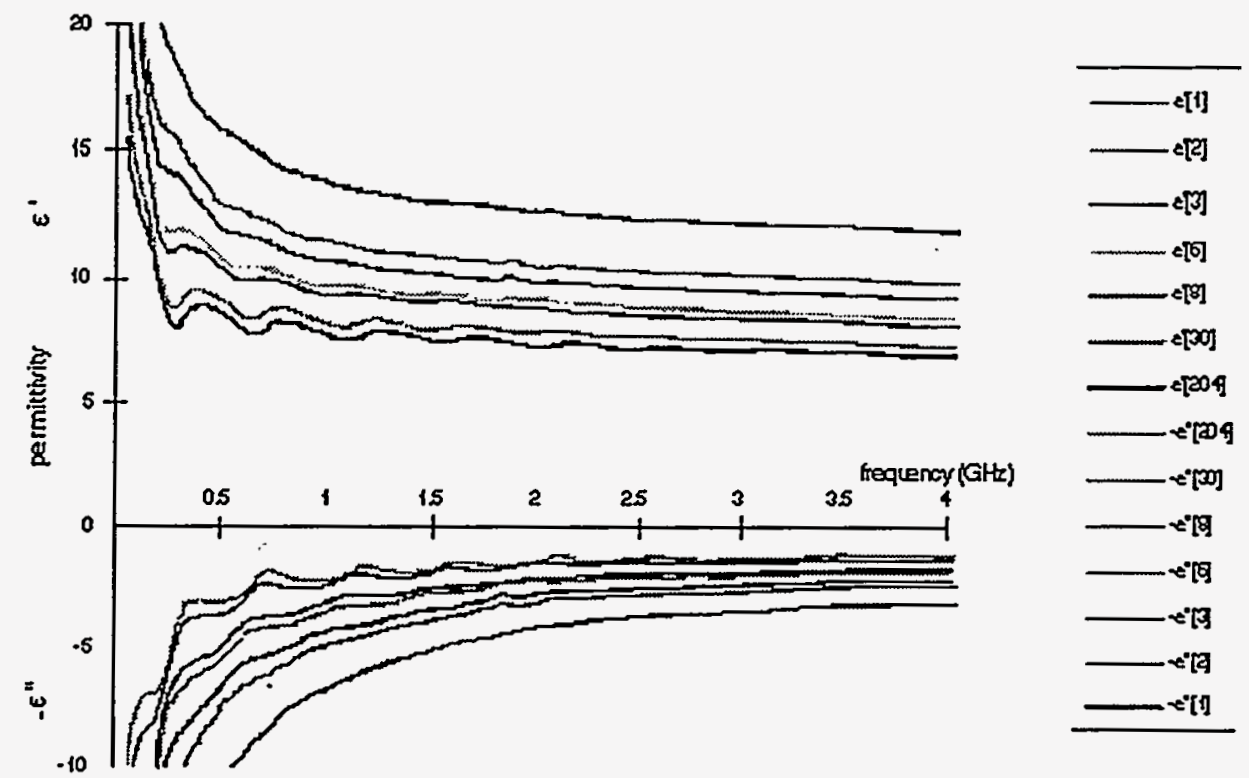

Figure 7. The relative complex permittivity of cement is shown versus the cure time. For clarity, the negative of the imaginary relative permittivity is shown in the graph. The legend $s$ indicators show the cure time in days (in brackets) in the same order as the graphs on the chart. Since the test sample used was small, the early time cure times were faster than what is expected for a large sample.

The EM concrete model included separate models for the cement and aggregate composing the concrete. The aggregate was modeled as homogeneous bodies in random orientations (see Fig 11). From the curves in Fig 7, the coefficients [2.11] were obtained for the cured sample and were used in the Lorentzian expansion

$$
F\left\{\sum_{\ell=1}^{\ell=\infty} \alpha_{\ell} e^{-\beta_{\ell} t} \sinh \left(\gamma_{\ell} t\right)\right\}=\frac{1}{2} \sum_{\ell=1}^{\ell=\infty} \frac{\alpha_{\ell}}{\beta_{\ell}-\gamma_{\ell}+j \omega}-\frac{\alpha_{\ell}}{\beta_{\ell}+\gamma_{\ell}+j \omega} \frac{(3)}{}
$$

to describe the frequency dependent complex permittivity. The discrete aggregate was modeled to simulate the high frequency experiment performed in the laboratory. The imaging algorithms [6] were able to image the discrete aggregate particles both from the modeled data and from the experimental data, thus a bulk model was unsuitable for this high frequency simulation.

\subsection{MODEL COMPẠRISONS}


The permittivity was used in $2 \mathrm{D}$ simulations to compare against "2D" experimental measurements made in the laboratory. The transmitting antenna was a ridged horn antenna while the receiving antenna was a Prodyne field probe. The experiment was limited to linear (horizontal) polarization in an attempt to simulate the 2D model.
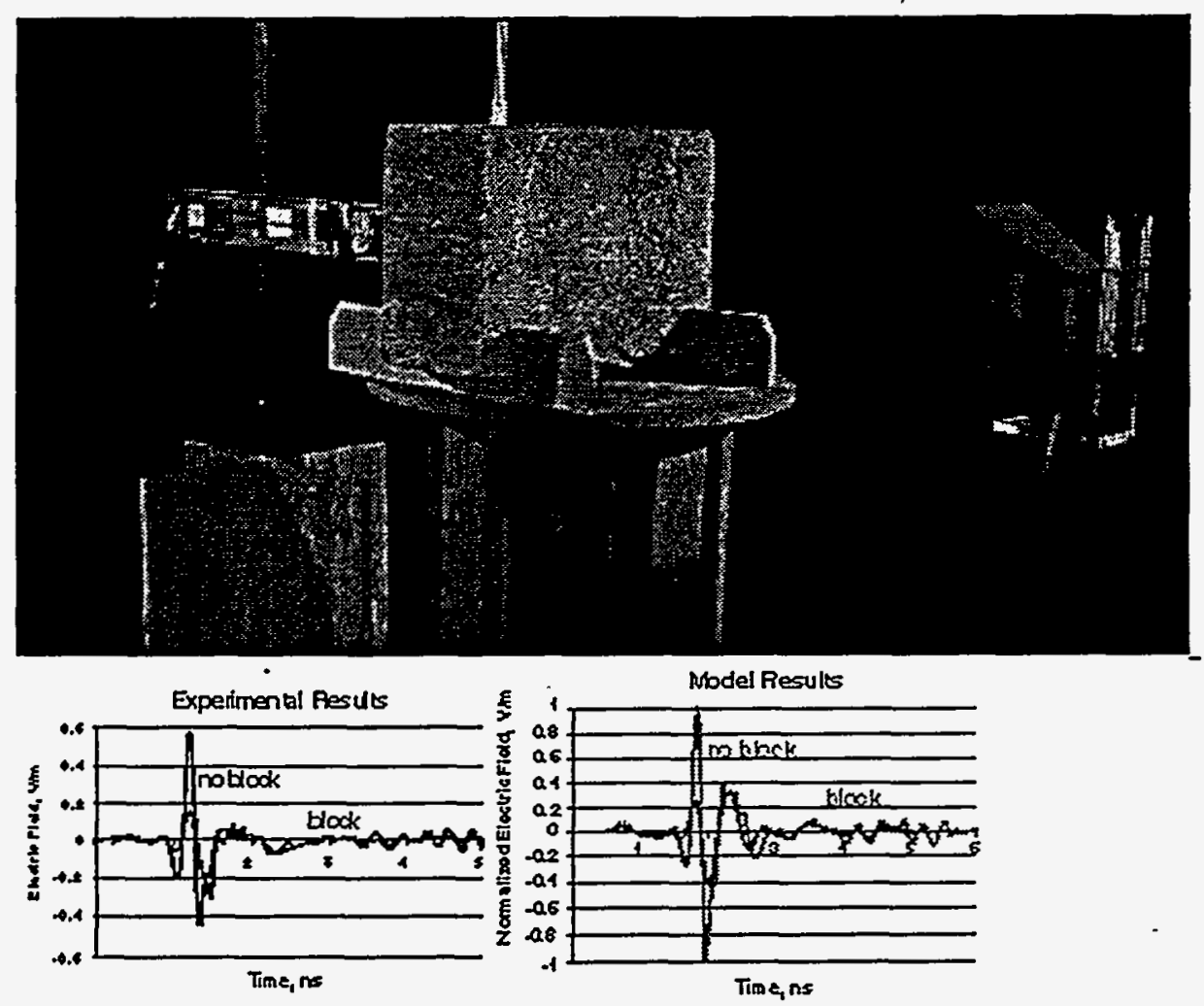

Figure 8. The experimental setup in the anechoic chamber is shown along with the experimental and modeling comparisons. Note the high intensity field at $2.5 \mathrm{~ns}$ in the model which is due to the grazing incidence off of the sides of the radiating boundary conditions.

There are two striking differences between the two waveforms above. One is due to the grazing incidence reflections off of the near-by radiating boundaries on the sides of the problem while the other is due to a 100ps temporal difference that occurs at the 2 ns point in the waveform. Since the exact placement of the aggregate is unknown in the concrete block above (but the duty cycle is known) then the 100ps difference is due to either the high frequency aggregate scattering or the missing dimension of the block. The exact cause was not investigated since only the $2 \mathrm{D}$ nature of the block was modeled.

\subsection{D Model comparison}

A broadband antenna model was used to compare the model with the experimental data. The antenna used in the model is shown in Fig 9 and is a model of the broadband doüble-ridged conical antennas. The time domain waveform for the modeled case is shown in Fig 10 for the 3D physical antenna. 


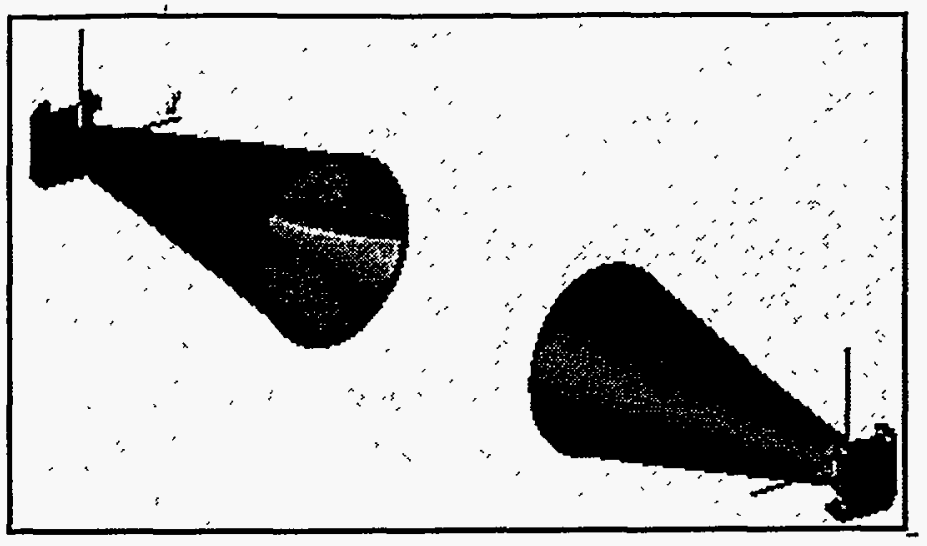

Figure 9. The antenna EM model used for the experimental comparison. This antenna is a dual ridged broadband conical hom antenna manufactured by EM Systems

Energy was launched from the feed region of the antenna towards the receiving antenna with an antenna aperture separation distance of one foot. The modeled and experimental waveforms are shown in Fig 10.

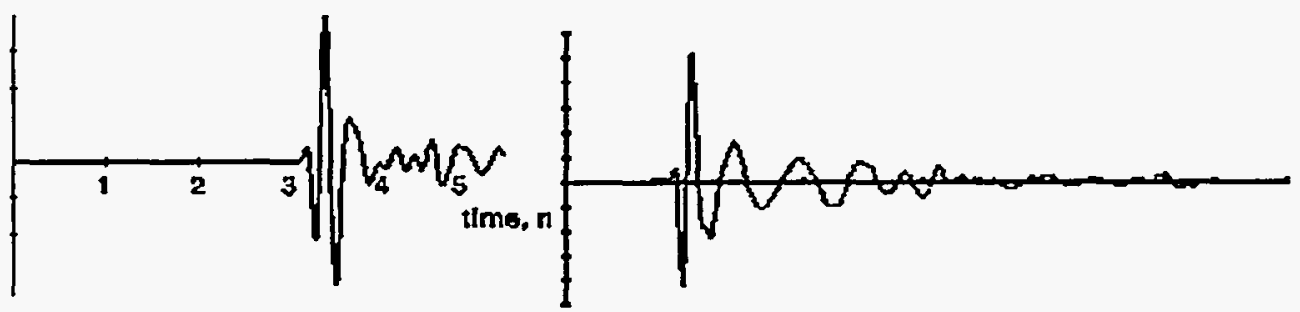

Figure 10. The time domain waveform for the 3D broadband antenna model shows the fast leading pulse and the later time ringing in the model (a) and the experiment (b). The relative time shift was due to the arbitrary time reference on the scope.

The early and mid-time effects agree well but the later time ringing does not due to the same reasons seen earlier in the $2 \mathrm{D}$ cases. In this case, the effects from the boundary occurred between $4 \mathrm{~ns}$ and $5 \mathrm{~ns}$ in the modeled result. Once again, small time shifts are preventing constructive addition of the wave at 3.5ns. Present models are correcting this problem by moving the energy launch plane. In general, it is necessary to move the boundary conditions away from the area of interest since high clutter geometries will invariably have off-normal energy propagation. This simple technique was used in the 3D models that will be seen later.

\subsection{D Concrete simulation}

Preliminary work for 3D geometries used a complex target and clutter geometry but a simple rectangular horn antenna model. Fig 11 shows a model using several rebar targets, three rebaric voids, a spherical air void, and a simple antenna. This rectangular waveguide fed horn antenna exhibited the fundamental ringing seen when 
sending a pulse through a section of rectangular waveguide and was expected. The wave packet was made short enough so that the ringing did not interfere with the imaging reconstruction.

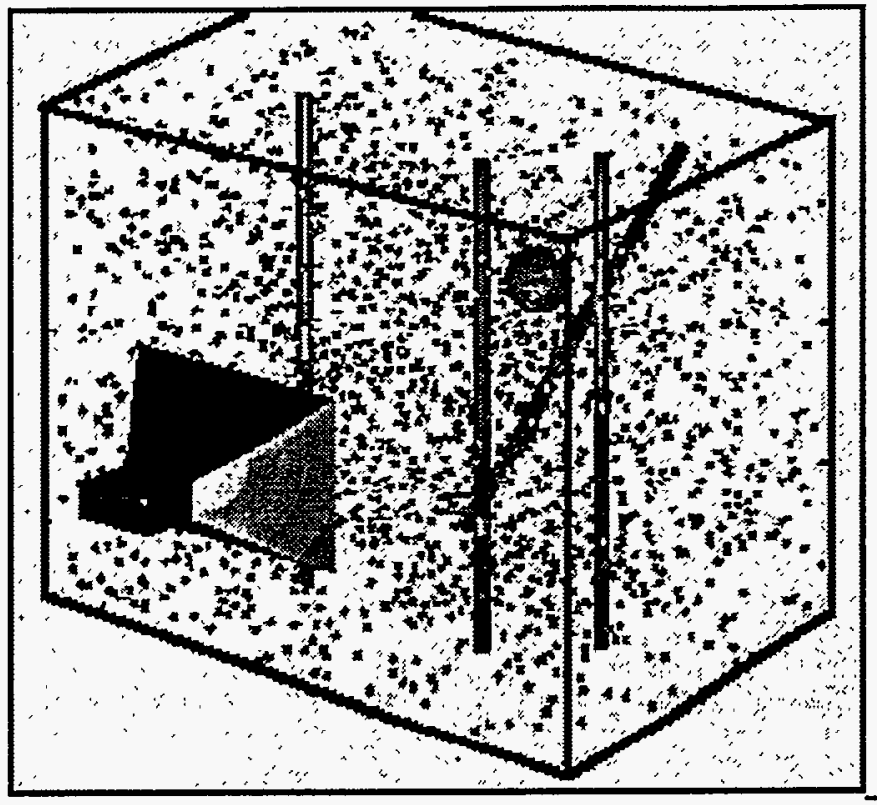

Figure 11 . The 3D geometry used for the concrete simulation containing randomly oriented cubical aggregate particles. The block has been outlined for illustration purposes and the cement removed to reveal the aggregate, rebars, rebaric voids, and spherical void.

The experimental geometry used for the 3D concrete slab verification is shown in Fig 12. In this case, broadband horn antennas were used in order to maintain the pulse fidelity. The energy was launched through the concrete test slab by the transmit antenna which was connected to the pulse generator. The receiver antenna was located 90 from the transmitter. The pulser generated a 400ps FWHM gaussian-like pulse which the antenna then differentiated. The receiving antenna was connected to a Tektronix CSA803 sampling scope to digitize the waveform and both antennas were offset from the concrete surface.

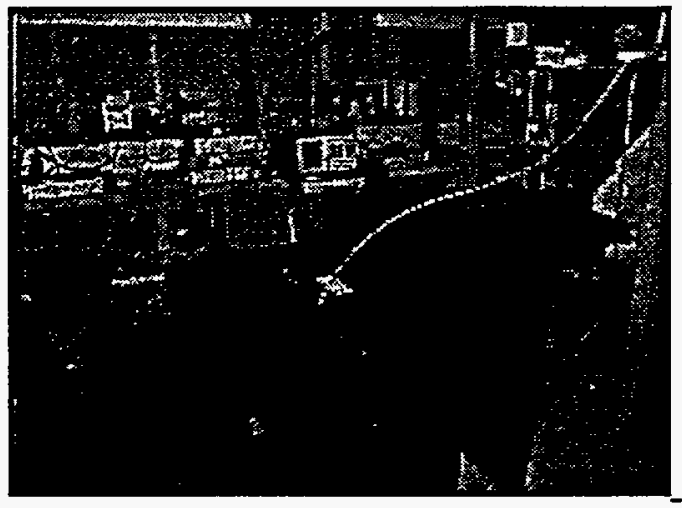



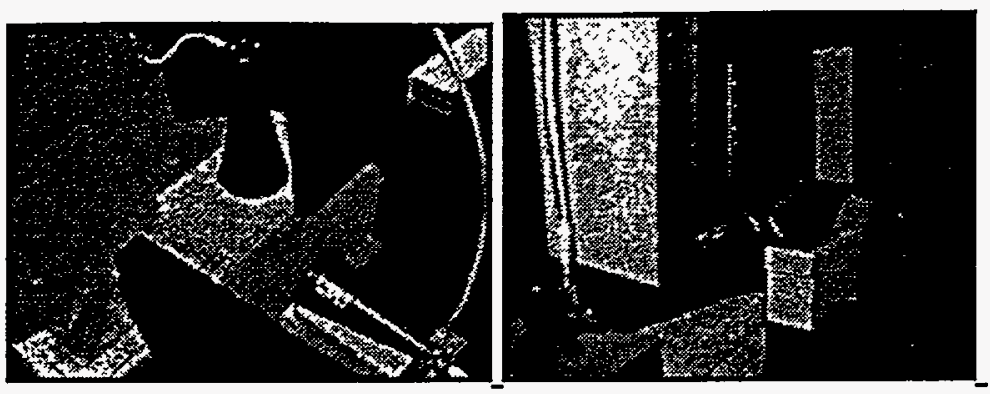

Figure 12. The concrete test slab, the conical horn antennas, the sampling scope, and the absorbing material are seen in this figure. The antennas were positioned so that the energy passed through the concrete; the antenna mutual coupling was included in the model.

\subsection{CONCLUSIONS}

The aggregate clutter models used were not sensitive to orientation as long as they remained small with respect to the pulse width. For clutter targets larger than rd of a pulsewidth in diameter, the clutter orientation is important. The packing density of the clutter for dense aggregate masses is more critical in $2 \mathrm{D}$ than $3 \mathrm{D}$ since diffraction occurs more readily in $3 \mathrm{D}$ since there are more diffraction surfaces. For dense clutter geometries, it is more important in $2 \mathrm{D}$ to insure that there is a propagation path through the model and is not practical when using high permittivity or highly conductive materials. These issues all trace back to the two-dimensional nature of the 2D models (i.e. circles are cylinders).

The 3D models used contained targets and randomly oriented clutter (the 3D mesher converted the randomly oriented cubes into multifaceted blobs). The imaging algorithms used were able to image the aggregate clutter particles and so a homogeneous effective material model was NOT suitable. This was confirmed using the experimental data7. This sizing limit greatly magnified the time required to do the 3D modeling but is very important because the aggregate clutter scatters, shadows, is diffractive, and is imaged as discrete particles. The goal of the modeling was to reduce the number of experiments required by accurately modeling the system. The imaging algorithms were not privy to this level of detail. Modeling was also used to control single elements in the simulations that are not possible with experiments.

One consistent problem with generating models using standard Yee based FDTD routines is the need for high quality boundary conditions. Unlike many other modeling applications where far field scattering, coupling, or cavity effects are being simulated, these GPIR applications are open air applications with complex scattering targets and near field antenna/material effects. These combine to make other types of boundary conditions more attractive such as Lindman or Liao. Also, observe that the 2nd order Mur boundaries that were used in the our 3D codes have problems with materials penetrating them (as do most boundary conditions) and thus air spaces are required which tend to act as wave channels for grazing incidence wave fields.

\subsection{Future plans}


A realistic ground model is being planned to include stratified earth, moisture gradations, rock effects, and possible bush root effects in $3 \mathrm{D}$ as part of some other projects. Some of these effects have already been demonstrated in $2 \mathrm{D}$. Also, the variability of the scanning antenna s flight path is being examined from a statistical standpoint to better simulate real world data acquisition conditions. This will allow for positional errors in the locatability of the antennas (all previous experiments used precisely known antenna locations).

\subsection{ACKNOWLEDGEMENTS}

Thanks go to Erik Johansson and Jeffrey Mast for their input on the imaging effort; to John Warhus for his hardware and pulser design efforts; to Carlos Avalle for his experimental efforts and analysis; and to Steve Pennock for his assistance with TSAR. The Ground Penetrating Imaging Radar Project (GPIR) was funded by the LLNL Microwave Pulse Power (Power Conversion) Thrust Area in conjunction with the Computational Electronics and Electromagnetics Thrust Area and other lab thrust areas to investigate the feasibility of designing an at-speed electromagnetic bridge deck inspection system. Contact: nelson18@llnl.gov

*This work was performed under the auspices of the U.S. Department of Energy by Lawrence Livermore National Laboratory under contract No. W-7405-Eng-48.

\subsection{REFERENCES}

1. G. J. Burke, A. J. Poggio, "Numerical Electromagnetic Code - Method of Moments,' Lawrence Livermore National Laboratory (UCID 18834), 1981.

2. J. DeFord, et. al., "Development and Applications of Dispersive Soft Ferrite Models for Time-Domain Simulation," $L L N L$ (UCRL-JC-109495), 1992.

3. S. D. Nelson, "Modeling of the [accelerator] Cavity," Lawrence Livermore National Laboratory, internal report, 1993.

4. R. R. McLeod, "Temporal scattering and response software," $L L N L$ (UCRL-MA 104861), June 1992.

5. S. D. Nelson and C. A. Avalle, "Electromagnetic Modeling and Experiments for Dispersive Media," Lawrence Livermore National Laboratory (from UCRL 53868-92), 1992.

6. E. Johansson and J. Mast, "Time and Frequency based 3D image reconstruction,' Second Government Workshop on GPR; Advanced Ground 
Penetrating Radar Technologies and Applications, (Sponsored by the US EPA [Region V] and the Ohio State University), October 26-28, 1993

7. S. D. Nelson, "Electromagnetic Modeling for Target Rich Embedded Environments," Lawrence Livermore National Laboratory (from UCRL 53868-93), 1993.

8. N. Madsen, S. Brandon, et. al., "Discrete Surface Integration -DSI3D," LLNL, March 1994.

9. J. A. Kong, Electromagnetic Wave Theory, John Wiley \& Sons (ISBN 0-471-52214-7), 1990.

10. C. Avalle, "Broadband Complex Permittivity Measurements of Cement," $L L N L$, in preparation, 1993.

11. K. S. Cole and R. H. Cole, "Dispersion and Absorption in Dielectrics," Journal of Chemical Physics, April 1941, pp 341-351.

If you have technical questions about this page, contact:

Scott D. Nelson,nelson18@llnl.gov

Last Modified: Aug 27, 1994

LLNL Disclaimer

UCRL-ID-?????? 